\title{
On the Effect of Vaccination, Screening and Treatment in Controlling Typhoid Fever Spread Dynamics: Deterministic and Stochastic Applications
}

\author{
Temitope Olu Ogunlade ${ }^{1}$, Oluwatayo Michael Ogunmiloro ${ }^{1}$, Segun Nathaniel Ogunyebi, \\ Grace Ebunoluwa Fatoyinbo ${ }^{1}$, Joshua Otonritse Okoro, ${ }^{2, *}$, Opeyemi Roselyn Akindutire ${ }^{3}$, \\ Omobolaji Yusuf Halid ${ }^{3}$, Adenike Oluwafunmilola Olubiyi ${ }^{3}$ \\ ${ }^{1}$ Department of Mathematics, Ekiti State University, Nigeria \\ ${ }^{2}$ Department of Physical Sciences, Landmark University, Nigeria \\ ${ }^{3}$ Department of Statistics, Ekiti State University, Nigeria
}

Received July 13, 2020; Revised September 11, 2020; Accepted September 29, 2020

\section{Cite This Paper in the following Citation Styles}

(a): [1] Temitope Olu Ogunlade, Oluwatayo Michael Ogunmiloro, Segun Nathaniel Ogunyebi, Grace Ebunoluwa Fatoyinbo, Joshua Otonritse Okoro, Opeyemi Roselyn Akindutire, Omobolaji Yusuf Halid, Adenike Oluwafunmilola Olubiyi, "On the Effect of Vaccination, Screening and Treatment in Controlling Typhoid Fever Spread Dynamics: Deterministic and Stochastic Applications," Mathematics and Statistics, Vol. 8, No. 6, pp. 621 - 630, 2020. DOI: 10.13189/ms.2020.080601.

(b): Temitope Olu Ogunlade, Oluwatayo Michael Ogunmiloro, Segun Nathaniel Ogunyebi, Grace Ebunoluwa Fatoyinbo, Joshua Otonritse Okoro Opeyemi Roselyn Akindutire, Omobolaji Yusuf Halid, Adenike Oluwafunmilola Olubiyi (2020). On the Effect of Vaccination, Screening and Treatment in Controlling Typhoid Fever Spread Dynamics: Deterministic and Stochastic Applications. Mathematics and Statistics, 8(6), 621 - 630. DOI: 10.13189/ms.2020.080601.

Copyright $\mathrm{C} 2020$ by authors, all rights reserved. Authors agree that this article remains permanently open access under the terms of the Creative Commons Attribution License 4.0 International License

\begin{abstract}
This work concerns a deterministic and stochastic model describing the transmission of typhoid fever infection in human host community, where the vaccination of susceptible births and immigrants as well as screening and treatment of carriers and infected individuals are considered in the model build - up. The well-posedness and computation of the basic reproduction number $R_{t y p}$ of the deterministic model are obtained and analysed. The deterministic model is further transformed into a stochastic model, where the drift and diffusion parts of the model are obtained, and the existence and uniqueness of the stochastic model are discussed. Numerical simulations involving the model parameters of $R_{t y p}$ showed that vaccination of susceptible births and influx of immigrants as well as screening and treatment of carriers and infected humans are effective in bringing the threshold $R_{t y p}\left(R_{t y p} \approx 0.7944\right)$ below 1 , and the results of other simulations suggest more health policies are to be implemented, as low $R_{\text {typ }}$ may not be guaranteed because vaccination wanes over time. In addition, the numerical simulations of the stochastic model equations describing the sub - population of human individuals in
\end{abstract}

the total human host community are carried out using the computational software MATLAB.

Keywords Reproduction Number, Existence and Uniqueness, Vaccination, Screening, Euler - Maruyama

\section{Introduction}

Typhoid fever is a dreadful disease of global health concern. The disease is contacted directly from person to person through the ingestion of contaminated food and water soiled with faeces. Humans and reservoirs serve as host to the causative bacteria Salmonella typhi. Typhoid fever endemic burden is high in the African and Asian continents, and some part of the western nations, where access to safe drinking water and food is inaccessible. According to the information available in the World Health Organization (WHO) fact sheet [17], 11 - 20 million humans become sick and between 128,000 - 161,000 
mortality cases were recorded every year. Clinical symptoms related to typhoid include fever, diarrhea, nausea, vomiting and abdominal pain. Antibiotics are effective drugs used in treating typhoid fever, though increasing resistance of typhoid strains to antibiotics makes the treatment complicated. Vaccines are readily available to prevent this disease, an injectable or oral vaccine in form of a capsule is administered. A new vaccine has recently been released since typhoid may mimic other clinical manifestations of diseases like malaria, dengue, hepatitis, etc. It is necessary that carriers be diagnosed using the conventional method and anti-body detection diagnosis. Typhoid is prevented through hygienic compliance, early administration of vaccine, accessibility to safe drinking water, treatment etc. Mathematical and stochastic models are good tools used in describing disease dynamics [4, 11]. Several good, descriptive and predictive deterministic and stochastic models have been derived to depict typhoid disease transmission. Adetunde [2], formulated a predictive model to describe the dynamical behavior of typhoid fever in the endemic regions of Kassena - Nankana district of upper east region of Ghana, where control measures were suggested to policy makers to reduce the spread of typhoid fever, see also [3]. Lauria et al, [8], formulated an optimization model for reducing typhoid cases in developing countries without increasing public spending. Mushayabasa [10], Watson and Edmund [16], investigated the impact of timely vaccination in controlling the spread of typhoid in typhoid disease dynamics, while Adeboye and Haruna [1] discussed the formulation and control of the co-infection of malaria and typhoid using the susceptible - infected - recovered approach. In addition, Omame et al. [13], formulated a stochastic model of typhoid fever, they investigated the existence and uniqueness of the model. Makinde, Getachew, and Tilahun [12] proposed a mathematical model of direct and indirect transmission of typhoid. The model was further extended into an optimal control problem and the cost-effective strategy was analyzed. Stephen and Nkuba [15] investigated the impact of education, vaccination and treatment as a form of effective control in minimizing typhoid fever infection through the formulation of a deterministic model, also, Aji, Aldila and Handari, Moathlhodi and Gosalamang [5, 9]. Peter et al. [14], employed the approximate method of variational iterative and Runge - Kutta fourth - order method to solve a system of ordinary differential equations describing typhoid dynamics. The approximate solutions of the model compare favorably with each other. Karunditu, Kimathi and Osman [7] formulated and a class of unprotected humans in the disease dynamics of typhoid fever, while Jegede [6] derived a stochastic dynamic of typhoid transmission, considering artificial immunity for disease through vaccination and natural immunity through recovery. In view of the related literature, our work differs by considering the formulation of a deterministic and model of typhoid fever dynamics to study the effect of vaccination for susceptible births and influx of immigrants and screening of carriers and treatment for screened and infected individuals. The combined intervention strategy is incorporated into the model in order to reduce and probably eliminate typhoid in human host community. Section 2 discusses the deterministic model derivation, analysis of the model and the computation of the basic reproduction number $R_{\text {typ }}$. Section 3 presents the transformation of the deterministic model into a stochastic model, where the transition probabilities, the drift and diffusion parts of the model are derived. Also, the existence and uniqueness of the derived stochastic model are discussed, while Section 4 presents the numerical simulations and discussion of results. Finally, Section 5 shows the conclusion of the work.

\section{Materials and Methods}

The model considered as described by Figurel is classified into sub - classes of the total human host population denoted $N_{\text {pop }}(t)$ as; Susceptible humans $S_{p}(t)$; Vaccinated humans $V_{c}(t)$; Infected humans $I_{f}(t)$; Carrier humans $C_{r}(t)$; Treated humans $T_{r}(t)$ and Recovered humans $R_{r}(t)$ at time $t>0$. The Susceptible humans sub-class $S_{p}(t)$ is generated by birth rate $\Lambda$, and reduced by the fraction of vaccinated births denoted by $(1-\rho)$. Also, this class is further increased by the influx of immigrants at the rate $\zeta$ and reduced by the proportion of vaccinated immigrants denoted, $(1-k)$. The effective infectious contact rate between a susceptible and infected individual is denoted, $\alpha_{1}$. Since vaccination wanes overtime, the rate at which vaccination wanes is denoted, $\tau$. Death due to natural causes not related to typhoid infection applicable to all sub - classes of human host population is denoted $\mu$. The transition rate from carrier to infected sub-class is denoted $\beta$, while $\eta_{1}$ denote the transition rate from infected to the treated sub - class and the typhoid related mortality rate is denoted, $\sigma$. Certain proportion of carrier humans underwent screening at the rate $\alpha_{2}$ and treatment at the rate $\delta$, while the proportion of those who weren't screened and treated is denoted by $(1-\delta) \alpha_{2}$. The transition rate from treated to recovered sub-class is denoted $\eta_{2}$, while the loss of immunity after recovery is denoted $e_{o}$. The assumptions guiding the model derivation are that birth and death rate is constant, vaccination of susceptible births and influx of immigrants are considered, there are recoveries through treatment and loss of immunity after recovery, while vaccination wanes overtime.

The inclusion of state variables and parameters describing the dynamics of typhoid fever infection leads to the system of deterministic first order ordinary differential equations given by

$$
\frac{d S_{p}}{d t}=(1-\rho) \Lambda-\left(\alpha_{1} I_{f}+\mu\right) S_{p}+(1-\kappa) \zeta+\tau V_{c}+e_{o} R_{r},
$$




$$
\begin{gathered}
\frac{\mathrm{dV}_{\mathrm{c}}}{\mathrm{dt}}=\rho \Lambda+\kappa \zeta-(\tau+\mu) \mathrm{V}_{\mathrm{c}}, \\
\frac{\mathrm{dI}_{\mathrm{f}}}{\mathrm{dt}}=\alpha_{1} \mathrm{~S}_{\mathrm{p}} \mathrm{I}_{\mathrm{f}}-\left(\beta+\eta_{1}+\sigma+\mu\right) \mathrm{I}_{\mathrm{f}} \\
\frac{\mathrm{dC}_{\mathrm{r}}}{\mathrm{dt}}=\beta \mathrm{I}_{\mathrm{f}}-(1-\delta) \alpha_{2} \mathrm{C}_{\mathrm{r}}-\mu \mathrm{C}_{\mathrm{r}} \\
\frac{\mathrm{dT}_{\mathrm{r}}}{\mathrm{dt}}=\eta_{1} \mathrm{I}_{\mathrm{f}}-\alpha_{2} \delta \mathrm{C}_{\mathrm{r}}-\left(\mu+\eta_{2}\right) \mathrm{T}_{\mathrm{r}} \\
\frac{\mathrm{dR}_{\mathrm{r}}}{\mathrm{dt}}=\eta_{2} \mathrm{~T}_{\mathrm{r}}-\left(\mu+\mathrm{e}_{\mathrm{o}}\right) \mathrm{R}_{\mathrm{r}}
\end{gathered}
$$

Subject to the initial conditions $S_{p}(0) \geq 0, V_{c}(0) \geq$ $0, I_{f}(0) \geq 0, C_{r}(0) \geq 0, T_{r}(0) \geq 0, R_{r}(0) \geq 0$

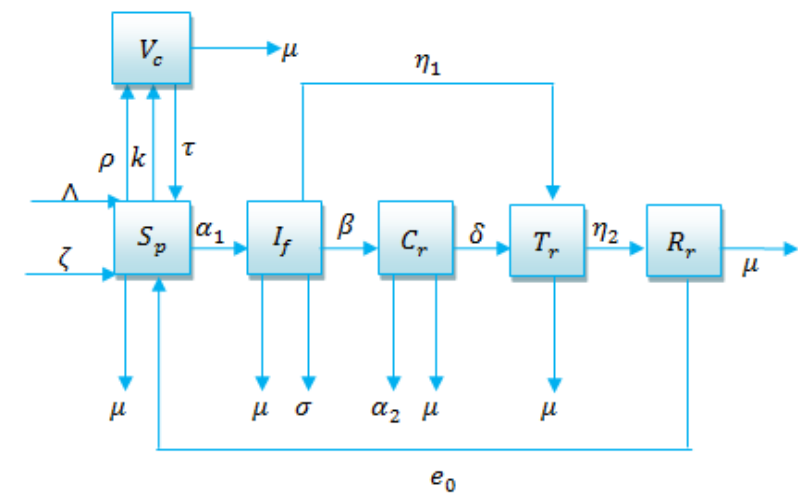

Figure 1. Diagrammatic representation of the infectious and recovery interactions among sub-classes of the total human host community

Figure 1 represents shows a diagrammatic flow of the disease progression.

\subsection{Model Analysis and Basic Reproduction Number $\boldsymbol{R}_{\text {typ }}$}

Theorem 1: A domain $\Omega$ exist where the solution space $\left(S_{p}, V_{c}, I_{f}, C_{r}, T_{r}, R_{r}\right)$ is contained and bounded.

proof: Given the solution space $\left(S_{p}, V_{c}, I_{f}, C_{r}, T_{r}, R_{r}\right)$ with positive initial data $S_{p}(0) \geq 0, V_{c}(0) \geq 0, I_{f}(0) \geq$ $0, C_{r}(0) \geq 0, T_{r}(0) \geq 0, R_{r}(0) \geq 0$, then

$$
\begin{gathered}
N_{\text {pop }}\left(S_{p}, V_{c}, I_{f}, C_{r}, T_{r}, R_{r}\right)=S_{p}(t)+V_{c}(t)+I_{f}(t)+ \\
C_{r}(t)+T_{r}(t)+R_{r}(t) .
\end{gathered}
$$

The addition of the derivative of $N_{\text {pop }}$ along the derivative of the model system (1) yields

$$
\begin{gathered}
N_{\text {pop }}^{\cdot}=(1-\rho) \Lambda+(1-\kappa) \zeta-\left(S_{p}+V_{c}+I_{f}+C_{r}+\right. \\
\left.T_{r}+R_{r}\right) \mu
\end{gathered}
$$

So that

$$
N_{\text {pop }}^{\cdot}=(1-\rho) \Lambda+(1-\kappa) \zeta-N_{\text {pop }} \mu
$$

The solution of (4) yields

$$
\begin{gathered}
N_{\text {pop }} \leq \frac{(1-\rho) \Lambda+(1-\kappa) \zeta}{\mu}(1-\exp (-\mu t))+N_{\text {pop }}\left(S_{p}+\right. \\
\left.V_{c}+I_{f}+C_{r}+T_{r}+R_{r}\right) \exp (-\mu t)
\end{gathered}
$$

As $t \rightarrow \infty$, taking the limit of both sides of (5) yields

$$
N_{\text {pop }} \leq \frac{(1-\rho) \Lambda+(1-\kappa) \zeta}{\mu}
$$

From (6), all solutions are contained in the domain $\Omega$ and are non - negative. The positive invariant domain exists and given by

$$
\Omega=\left\{\left(S_{p}, V_{c}, I_{f}, C_{r}, T_{r}, R_{r}\right) \in \mathbb{R}^{6+}: N_{p o p} \leq \frac{(1-\rho) \Lambda+(1-\kappa) \zeta}{\mu}\right\}
$$

Hence, (1) is well - defined and reasonable in the sense of typhoid dynamics. The trivial fixed point solution does not exist as long as the recruitment of birth of susceptible term and influx terms exist. Therefore, the typhoid - free fixed point solutions i.e., when the system is free of typhoid infection is given by

$$
\left(S_{p}, V_{c}, I_{f}, C_{r}, T_{r}, R_{r}\right)=\left(\frac{(1-\rho) \Lambda+(1-\kappa) \zeta}{\mu}, \frac{\rho \Lambda+\kappa \zeta}{\tau+\mu}, 0,0,0,0\right) .
$$

The basic reproduction number $\left(R_{\text {typ }}\right)$ of this model system is the rate of transmissibility of typhoid infection in a largely susceptible population in the course of infection. The next generation matrix method is employed to obtain the threshold, see $[12,15]$. The two typhoid infected class $I_{f}$ and $C_{r}$ is linearized around the typhoid - free fixed point solution to give the basic reproduction number of model system (1) as

$$
R_{t y p}=\frac{\alpha_{1} \Lambda(1-\rho)+(1-\kappa) \zeta}{\mu\left(\beta+\eta_{1}+\sigma+\mu\right)}
$$

In (9), $R_{\text {typ }}$ is called a vaccination controlled reproduction number, where births of susceptible recruited and influx of immigrants are vaccinated, where $\rho>1-\frac{1}{R_{t y p}}$ and $\kappa>1-\frac{1}{R_{t y p}}$ leads to a herd immunity level. The parameter $\alpha_{1}$ denoted typhoid transmission rate per single infective, while each infectious individual spends on average $\frac{1}{\beta+\eta_{1}}$ time units in their class. The period of typhoid infection is reduced due to natural and typhoid related mortality. In this work, $R_{\text {typ }} \approx 0.744$, this is less than 1 .

However, $R_{t y p}$ can be greater than 1 , if vaccination wanes overtime and there is non-compliance to other public health measures. The impact of the model parameters involved in the computation of $R_{t y p}$ is analyzed in section 4 . 
Table 1. Parameter descriptions of the model describing Typhoid dynamics

\begin{tabular}{|c|c|c|c|}
\hline Parameters & Descriptions & Values & Sources \\
\hline$\Lambda$ & Recruitment of susceptible births & 0.83681 & {$[13]$} \\
\hline$\rho$ & Fraction of vaccinated susceptible & 0.9 & {$[8]$} \\
\hline$\alpha_{1}$ & Effective infectious contact rate & 0.0002 & {$[13]$} \\
\hline$\zeta$ & Infected immigrants & 0.027 & Assumed \\
\hline$\mu$ & Natural death rate & 0.02041 & {$[17]$} \\
\hline$\kappa$ & Fraction of vaccinated immigrants & 0.09 & Assumed \\
\hline$\tau$ & Vaccination waning rate & 0.33 & {$[13]$} \\
\hline$e_{0}$ & Loss of immunity & 0.33 & {$[14]$} \\
\hline$\eta_{1}$ & Transition rate from infected to carrier class & 0.5 & {$[3]$} \\
\hline$\alpha_{2}$ & Transition rate from infected to treated class & 0.096 & {$[12]$} \\
\hline$\delta$ & Screened carriers & 0.2 & Assumed \\
\hline$\eta_{2}$ & Treated carriers & 0.2115 & {$[9]$} \\
\hline
\end{tabular}

\section{Transformation of the Deterministic Model (1) into a Stochastic Model}

Table 2. Transition probabilities

\begin{tabular}{|c|c|c|}
\hline Changes & Probabilities & Event \\
\hline$\left[\begin{array}{llllll}1 & 0 & 0 & 0 & 0 & 0\end{array}\right]^{\mathrm{T}}$ & $\mathrm{P}_{1}=\Lambda \Delta \mathrm{t}$ & Susceptible Birth \\
\hline$\left[\begin{array}{llllll}1 & 0 & 0 & 0 & 0 & 0\end{array}\right]^{\mathrm{T}}$ & $\mathrm{P}_{2}=\zeta \Delta \mathrm{t}$ & Influx of immigrants \\
\hline$\left[\begin{array}{llllll}-1 & 0 & 1 & 0 & 0 & 0\end{array}\right]^{\mathrm{T}}$ & $\mathrm{P}_{3}=\alpha_{1} \mathrm{~S}_{\mathrm{p}} \mathrm{I}_{\mathrm{f}} \Delta \mathrm{t}$ & $\begin{array}{l}\text { Effective infectious contact } \\
\text { rate }\end{array}$ \\
\hline$\left[\begin{array}{llllll}-1 & 1 & 0 & 0 & 0 & 0\end{array}\right]^{\mathrm{T}}$ & $\mathrm{P}_{4}=\kappa \zeta \Delta \mathrm{t}$ & Vaccinated immigrants \\
\hline$\left[\begin{array}{llllll}-1 & 1 & 0 & 0 & 0 & 0\end{array}\right]^{\mathrm{T}}$ & $P_{5}=\rho \Lambda \Delta t$ & Vaccinated susceptible \\
\hline$\left[\begin{array}{llllll}-1 & 0 & 0 & 0 & 0 & 0\end{array}\right]^{\mathrm{T}}$ & $\mathrm{P}_{6}=\mu \mathrm{S}_{\mathrm{p}} \Delta \mathrm{t}$ & Natural death of susceptible \\
\hline$\left[\begin{array}{llllll}1 & -1 & 0 & 0 & 0 & 0\end{array}\right]^{\mathrm{T}}$ & $\mathrm{P}_{7}=\tau \mathrm{V}_{\mathrm{c}} \Delta \mathrm{t}$ & Vaccination waning rate \\
\hline$\left[\begin{array}{llllll}0 & -1 & 0 & 0 & 0 & 0\end{array}\right]^{\mathrm{T}}$ & $\mathrm{P}_{8}=\mu \mathrm{V}_{\mathrm{c}} \Delta \mathrm{t}$ & $\begin{array}{l}\text { Natural death rate of } \\
\text { vaccinated }\end{array}$ \\
\hline 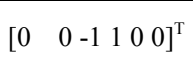 & $\mathrm{P}_{9}=\beta \mathrm{I}_{\mathrm{f}} \Delta \mathrm{t}$ & $\begin{array}{l}\text { Transition rate of infected to } \\
\text { carrier class }\end{array}$ \\
\hline 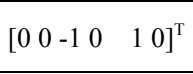 & $\mathrm{P}_{10}=\eta_{1} \mathrm{I}_{\mathrm{f}} \Delta \mathrm{t}$ & $\begin{array}{l}\text { Transition rate of infected to } \\
\text { treated class }\end{array}$ \\
\hline$\left[\begin{array}{llllll}0 & 0 & -1 & 0 & 0 & 0\end{array}\right]^{\mathrm{T}}$ & $\mathrm{P}_{11}=\sigma \mathrm{I}_{\mathrm{f}} \Delta \mathrm{t}$ & Death due to typhoid infection \\
\hline$\left[\begin{array}{llllll}0 & 0 & -1 & 0 & 0 & 0\end{array}\right]^{\mathrm{T}}$ & $\mathrm{P}_{12}=\mu \mathrm{I}_{\mathrm{f}} \Delta \mathrm{t}$ & $\begin{array}{l}\text { Natural death rate of } \\
\text { infectives }\end{array}$ \\
\hline$\left[\begin{array}{llllll}0 & 0 & 0 & -1 & 1 & 0\end{array}\right]^{\mathrm{T}}$ & $\mathrm{P}_{13}=\delta \alpha_{2} \mathrm{C}_{\mathrm{r}} \Delta \mathrm{t}$ & Treated carriers \\
\hline$\left[\begin{array}{llllll}0 & 0 & 0 & -1 & 0 & 0\end{array}\right]^{\mathrm{T}}$ & $\mathrm{P}_{14}=\alpha_{2} \mathrm{C}_{\mathrm{r}} \Delta \mathrm{t}$ & Screened carriers \\
\hline$\left[\begin{array}{llllll}0 & 0 & 0 & -1 & 0 & 0\end{array}\right]^{\mathrm{T}}$ & $\mathrm{P}_{15}=\mu \mathrm{C}_{\mathrm{r}} \Delta \mathrm{t}$ & Natural death rate of carriers \\
\hline$\left[\begin{array}{llllll}0 & 0 & 0 & 0 & -1 & 1\end{array}\right]^{\mathrm{T}}$ & $P_{16}=\eta_{2} T_{r} \Delta t$ & $\begin{array}{l}\text { Transition rate of treated to } \\
\text { recovered class }\end{array}$ \\
\hline$\left[\begin{array}{llllll}0 & 0 & 0 & 0 & -1 & 0\end{array}\right]^{\mathrm{T}}$ & $\mathrm{P}_{17}=\mu \mathrm{T}_{\mathrm{r}} \Delta \mathrm{t}$ & $\begin{array}{l}\text { Natural death rate of treated } \\
\text { individuals }\end{array}$ \\
\hline$\left[\begin{array}{llllll}0 & 0 & 0 & 0 & 0 & -1\end{array}\right]^{\mathrm{T}}$ & $\mathrm{P}_{18}=\mu \mathrm{R}_{\mathrm{r}} \Delta \mathrm{t}$ & $\begin{array}{l}\text { Natural death rate of } \\
\text { recovered individuals }\end{array}$ \\
\hline$\left[\begin{array}{llllll}1 & 0 & 0 & 0 & 0 & -1\end{array}\right]^{\mathrm{T}}$ & $\mathrm{P}_{19}=\mathrm{e}_{\mathrm{o}} \mathrm{R}_{\mathrm{r}} \Delta \mathrm{t}$ & $\begin{array}{l}\text { Loss of immunity in } \\
\text { recovered individuals }\end{array}$ \\
\hline
\end{tabular}

Here, the interest is changing the parameters in model system (1) into random variables, where the randomness is incorporated into the stochastic model. The stochastic model consists of the drift or deterministic parts and diffusion or stochastic parts. In order to obtain the mean and the variance of the model, the transition probabilities obtained in model system (1) are presented in Table 2 .

\subsection{The Stochastic Model Equations}

As developed by Allen [4], the stochastic model equations are given by

$$
\begin{gathered}
d \vec{X}=\vec{f}(\mathrm{t}, \vec{X}(\mathrm{t})) d t+G(\mathrm{t}, \vec{X}(\mathrm{t})) d \vec{W}(\mathrm{t}) \\
\vec{X}(0)=\left[\mathrm{X}_{1}(0), \mathrm{X}_{2}(0), \mathrm{X}_{3}(0), \mathrm{X}_{4}(0), \mathrm{X}_{5}(0), \mathrm{X}_{6}(0)\right]^{\mathrm{T}}
\end{gathered}
$$

Where the drift vector is defined as

$$
\vec{f}=\sum_{j=1}^{19} p_{j} \vec{\lambda}_{j}
$$

Where $\vec{\lambda}_{j}$ and $p_{j}$ are the random changes and transition probabilities respectively. Applying (10) and (11) to the model system (1) yields the drift vector $\vec{f}$ given by

$$
\vec{f}=\left(\begin{array}{c}
(1-\rho) \Lambda-\left(\alpha_{1} \mathrm{I}_{\mathrm{f}}+\mu\right) \mathrm{S}_{\mathrm{p}}+(1-\kappa) \zeta+\tau \mathrm{V}_{\mathrm{c}}+\mathrm{e}_{\mathrm{o}} \mathrm{R}_{\mathrm{r}}, \\
\rho \Lambda+\kappa \zeta-(\tau+\mu) \mathrm{V}_{\mathrm{c}}, \\
\alpha_{1} \mathrm{~S}_{\mathrm{p}} \mathrm{I}_{\mathrm{f}}-\left(\beta+\eta_{1}+\sigma+\mu\right) \mathrm{I}_{\mathrm{f}} \\
\beta \mathrm{I}_{\mathrm{f}}-(1-\delta) \alpha_{2} \mathrm{C}_{\mathrm{r}}-\mu \mathrm{C}_{\mathrm{r}} \\
\eta_{1} \mathrm{I}_{\mathrm{f}}-\alpha_{2} \delta \mathrm{C}_{\mathrm{r}}-\left(\mu+\eta_{2}\right) \mathrm{T}_{\mathrm{r}} \\
\eta_{2} \mathrm{~T}_{\mathrm{r}}-\left(\mu+\mathrm{e}_{\mathrm{o}}\right) \mathrm{R}_{\mathrm{r}} .
\end{array}\right)
$$

The diffusion matrix $\mathrm{G}$ in (10) has the entries $\lambda_{i, j} p_{j}^{1 / 2}$, Where $\lambda_{i, j}$ and $p_{j}(\mathrm{i}=1, \ldots 6, \mathrm{j}=1, . .19)$ are the 
components of the random changes and transition probabilities respectively. Also,

$$
\vec{W}(\mathrm{t})=\left[\mathrm{W}_{1}(\mathrm{t}), \mathrm{W}_{2}(\mathrm{t}), \mathrm{W}_{3}(\mathrm{t}), \ldots, \mathrm{W}_{19}(\mathrm{t})\right]^{\mathrm{T}}
$$

This is a vector of nineteen independent Wiener processes.

Considering $\vec{f}(\mathrm{t}, \vec{X}(\mathrm{t}))$ as the drift part and $G(\mathrm{t}, \vec{X}(\mathrm{t}))$ as the diffusion part where

$\vec{f}(\mathrm{t}, \vec{X}(\mathrm{t}))=\frac{E \Delta x}{\Delta t}$ and $G(\mathrm{t}, \vec{X}(\mathrm{t}))=V^{1 / 2}=\sqrt{\frac{E\left[\Delta x \Delta x^{T}\right]}{\Delta t}}$

Then $E \Delta x=\sum_{i=1}^{19} p_{i}(\Delta x)_{\mathrm{i}} \Delta t$, so that

$$
\mathrm{E}[\Delta \mathrm{x}]=\left(\begin{array}{c}
\mathrm{P}_{1}+\mathrm{P}_{2}-\mathrm{P}_{3}-\mathrm{P}_{4}-\mathrm{P}_{5}-\mathrm{P}_{6}+\mathrm{P}_{7}+\mathrm{P}_{19} \\
\mathrm{P}_{4}+\mathrm{P}_{5}-\mathrm{P}_{7}-\mathrm{P}_{8} \\
\mathrm{P}_{3}-\mathrm{P}_{9}-\mathrm{P}_{10}-\mathrm{P}_{11}-\mathrm{P}_{12} \\
\mathrm{P}_{9}-\mathrm{P}_{13}-\mathrm{P}_{14}-\mathrm{P}_{15} \\
\mathrm{P}_{10}+\mathrm{P}_{13}-\mathrm{P}_{16}-\mathrm{P}_{17} \\
\mathrm{P}_{16}-\mathrm{P}_{18}-\mathrm{P}_{19}
\end{array}\right)
$$

And $E\left[\Delta x \Delta x^{T}\right]=\sum_{i=1}^{19} p_{i}(\Delta x)_{\mathrm{i}} \Delta t^{\mathrm{T}} \Delta$, where

$$
\mathrm{E}\left[\Delta x \Delta x^{T}\right]=\left(\begin{array}{c}
\mathrm{P}_{1}+\mathrm{P}_{2}+\mathrm{P}_{6} \\
\mathrm{P}_{8} \\
\mathrm{P}_{11}+\mathrm{P}_{12} \\
\mathrm{P}_{14}+\mathrm{P}_{15} \\
\mathrm{P}_{17} \\
\mathrm{P}_{18}
\end{array}\right)
$$

From (12) - (15), the transformed stochastic model equations are given by:

$$
\begin{aligned}
& \dot{S}_{P}=(1-\mathrm{Q}) \Lambda-\alpha_{1} \mathrm{~S}_{\mathrm{p}} \mathrm{I}_{\mathrm{f}}+(1-\kappa) \zeta-\mu \mathrm{S}_{\mathrm{p}}+\tau \mathrm{V}_{\mathrm{c}}+\mathrm{e}_{\mathrm{o}} \mathrm{R}_{\mathrm{r}}+ \\
& \sqrt{\left(P_{1}+P_{2}-P_{3}-P_{4}-P_{5}-P_{6}+P_{7}+P_{19}\right) W_{1}} \\
& +\sqrt{P_{1} W_{1}}+\sqrt{P_{2} W_{2}}+\sqrt{P_{6} W_{6}} \\
& \dot{V}_{C}=\rho \Lambda+\kappa \zeta-\tau \mathrm{V}_{\mathrm{c}}-\mu \mathrm{V}_{\mathrm{c}}+\sqrt{\left(P_{4}+P_{5}-P_{7}-P_{8}\right) W_{2}} \\
& +\sqrt{P_{8} W_{8}} \\
& \begin{array}{c}
\dot{I_{f}}=\alpha 1 \text { SpIf }-(\beta+\eta 1+\sigma+\mu) \text { If }+ \\
\sqrt{\left(P_{3}-P_{9}-P_{10}-P_{11}-P_{12}\right) W_{3}}+\sqrt{P_{11} W_{11}}+
\end{array} \\
& \sqrt{P_{12} W_{12}} \\
& \frac{\dot{C}_{r}=\beta \mathrm{If}-(1-\delta) \alpha 2 \mathrm{Cr}-\mu \mathrm{Cr}+}{\sqrt{\left(P_{9}-P_{13}-P_{14}-P_{15}\right) W_{4}}+\sqrt{P_{14} W_{14}}+\sqrt{P_{15} W_{15}}} \\
& \begin{array}{c}
\dot{T}_{r}=\eta 1 \text { If }-\alpha 2 \delta \mathrm{Cr}-\mu \mathrm{Tr}-\eta 2 \mathrm{Tr}+ \\
\sqrt{\left(P_{10}+P_{13}-P_{16}-P_{17}\right) W_{5}}+\sqrt{P_{17} W_{17}}
\end{array} \\
& \dot{R_{r}}=\eta 2 \mathrm{Tr}-\mu \mathrm{Rr}-\mathrm{e} 0 \mathrm{Rr}+\sqrt{\left(P_{16}-P_{15}-P_{19}\right) W_{6}}+ \\
& \sqrt{P_{18} W_{18}}
\end{aligned}
$$

Under the initial conditions $\mathrm{S}_{\mathrm{p}}(0) \geq 0, \mathrm{~V}_{\mathrm{c}}(0) \geq 0, \mathrm{I}_{\mathrm{f}}(0) \geq 0$, $\mathrm{C}_{\mathrm{r}}(0) \geq 0, \mathrm{R}_{\mathrm{r}}(0) \geq 0$.

\subsection{Existence and Uniqueness of the Stochastic Model \\ (16)}

Assuming that the coefficients in the following system of differential equations

$$
\mathrm{d} X_{1}^{i}=\mathrm{a}_{1}\left(\mathrm{t}, \mathrm{X}_{\mathrm{t}}\right) d t+\sum_{i=1}^{n} \sum_{j=1}^{m} b_{i j}\left(\mathrm{t}, \mathrm{X}_{\mathrm{t}}\right) d W_{t}^{1}
$$

where

$$
\begin{gathered}
\mathrm{X}_{\mathrm{t}}=\left(X_{t}^{1}, X_{t}^{2}, \ldots X_{t}^{n}\right)^{\mathrm{T}} \\
\mathrm{W}_{\mathrm{t}}=\left(W_{t}^{1}, W_{t}^{2}, \ldots W_{t}^{m}\right)^{\mathrm{T}}
\end{gathered}
$$

$a_{\mathrm{i}}\left(t, X_{t}\right)$ is an n-dimensional vectors with entries $\mathrm{a}_{1}(t, x)$ and $\mathrm{b}_{\mathrm{ij}}(t, x)$ is an $\mathrm{n} \times \mathrm{m}$ matrix with entries $\mathrm{b}_{\mathrm{ij}}(t, x)$ satisfy the following Lipschitz and growth conditions for some constant $k<\infty$, and for all $\mathrm{t} \in \mathrm{R}$ and $\mathrm{x}, \mathrm{y} \in \mathrm{R}^{\mathrm{n}}$ with the following

$$
\begin{gathered}
\left\|a_{i}(\mathrm{t}, \mathrm{x})-a_{i}(t, y)\right\| \leq \mathrm{k}\|x-y\|, \\
\left\|b_{i j}(t, x)-b_{i j}(t, y)\right\| \leq \mathrm{k}\|x-y\|, \\
\left\|a_{i}(t, x)\right\| \leq \mathrm{k}\|x\|, \\
\left\|b_{i j}(t, x)\right\| \leq \mathrm{k}\|x\|, \\
\|b\|=\sqrt{\sum_{i=1}^{n} \sum_{j=1}^{m} b_{i j}(x)^{2}} \\
\|a\|=\sqrt{\sum_{i=1}^{n} a_{i}(x)^{2}}
\end{gathered}
$$

Then for each $x \in \mathrm{R}^{\mathrm{n}}$ there is a unique solution to the system of stochastic differential equations (16) such that $X$ $=x$. The following changes are made from (16) so that

$$
\begin{gathered}
f_{l}=(1-\rho) \Lambda-\alpha_{1} \mathrm{~S}_{\mathrm{p}} \mathrm{I}_{\mathrm{f}}+(1-\kappa) \zeta-\mu \mathrm{S}_{\mathrm{p}}+\tau \mathrm{V}_{\mathrm{c}}+\mathrm{e}_{\mathrm{o}} \mathrm{R}_{\mathrm{r}} \\
f_{2}=\rho \Lambda+\kappa \zeta-\tau \mathrm{V}_{\mathrm{c}}-\mu \mathrm{V}_{\mathrm{c}} \\
f_{3}=\alpha_{1} \mathrm{~S}_{\mathrm{p}} \mathrm{I}_{\mathrm{f}}-\left(\beta+\eta_{1}+\sigma+\mu\right) \mathrm{I}_{\mathrm{f}} \\
f_{4}=\beta \mathrm{I}_{\mathrm{f}}-(1-\delta) \alpha_{2} \mathrm{C}_{\mathrm{r}}-\mu \mathrm{C}_{\mathrm{r}} \\
f_{5}=\eta_{1} \mathrm{I}_{\mathrm{f}}-\alpha_{2} \delta \mathrm{C}_{\mathrm{r}}-\mu \mathrm{T}_{\mathrm{r}}-\eta_{2} \mathrm{~T}_{\mathrm{r}} \\
f_{6}=\eta_{2} \mathrm{~T}_{\mathrm{r}}-\mu \mathrm{R}_{\mathrm{r}}-\mathrm{e}_{\mathrm{o}} \mathrm{R}_{\mathrm{r}}
\end{gathered}
$$

Then there exists a constant $\mathrm{M}>0$ such that

$$
\begin{gathered}
\left|\frac{\partial f_{1}}{\partial s_{p}}\right|=\left|\alpha_{1} I_{f}+\mu\right| \leq \mathrm{M},\left|\frac{\partial f_{1}}{\partial V_{c}}\right|=|\tau| \leq \mathrm{M},\left|\frac{\partial f_{1}}{\partial I_{f}}\right|=\left|\alpha_{1} \mathrm{~S}_{\mathrm{p}}\right| \\
\leq \mathrm{M},\left|\frac{\partial f_{1}}{\partial C_{r}}\right|=\left|\frac{\partial f_{1}}{\partial T_{r}}\right|=0,\left|\frac{\partial f_{1}}{\partial R_{r}}\right|=\left|\mathrm{e}_{0}\right| \leq M, \\
\left|\frac{\partial f_{2}}{\partial s_{p}}\right|=\left|\frac{\partial f_{2}}{\partial I_{f}}\right|=\left|\frac{\partial f_{2}}{\partial C_{r}}\right|=\left|\frac{\partial f_{2}}{\partial T_{r}}\right|=\left|\frac{\partial f_{2}}{\partial R_{r}}\right|=0,\left|\frac{\partial f_{2}}{\partial V_{c}}\right|= \\
|\tau+\mu| \leq \mathrm{M},
\end{gathered}
$$

$\left|\frac{\partial f_{3}}{\partial s_{p}}\right|=\left|\alpha_{1} I_{f}\right| \leq \mathrm{M},\left|\frac{\partial f_{3}}{\partial I_{f}}\right|=\left|\beta+\eta_{1}+\sigma+\mu\right| \leq \mathrm{M}$,

$$
\left|\frac{\partial f_{3}}{\partial V_{c}}\right|=\left|\frac{\partial f_{3}}{\partial C_{r}}\right|=\left|\frac{\partial f_{3}}{\partial T_{r}}\right|=\left|\frac{\partial f_{3}}{\partial R_{r}}\right|=0,
$$

$\left|\frac{\partial f_{4}}{\partial s_{p}}\right|=\left|\frac{\partial f_{4}}{\partial V_{c}}\right|=\left|\frac{\partial f_{4}}{\partial T_{r}}\right|=\left|\frac{\partial f_{4}}{\partial R_{r}}\right|=0,\left|\frac{\partial f_{4}}{\partial I_{f}}\right|=|\beta| \leq \mathrm{M}$, $\left|\frac{\partial f_{4}}{\partial C_{r}}\right|=\left|(1-\delta) \alpha_{2}+\mu\right| \leq \mathrm{M}$,

$$
\begin{gathered}
\left|\frac{\partial f_{5}}{\partial s_{p}}\right|=\left|\frac{\partial f_{5}}{\partial V_{c}}\right|=\left|\frac{\partial f_{5}}{\partial R_{r}}\right|=0,\left|\frac{\partial f_{5}}{\partial I_{f}}\right|=\left|\eta_{1}\right| \leq \mathrm{M},\left|\frac{\partial f_{5}}{\partial C_{r}}\right|= \\
\left|\alpha_{2} \delta\right| \leq \mathrm{M},\left|\frac{\partial f_{5}}{\partial T_{r}}\right|=\left|\mu+\eta_{2}\right| \leq \mathrm{M}
\end{gathered}
$$

$$
\begin{gathered}
\left|\frac{\partial f_{6}}{\partial s_{p}}\right|=\left|\frac{\partial f_{6}}{\partial V_{c}}\right|=\left|\frac{\partial f_{6}}{\partial I_{f}}\right|=\left|\frac{\partial f_{6}}{\partial c_{r}}\right|=0,\left|\frac{\partial f_{6}}{\partial T_{r}}\right|=\left|\eta_{2}\right| \leq \mathrm{M}, \\
\left|\frac{\partial f_{6}}{\partial R_{r}}\right|=\left|\mathrm{e}_{0}+\mu\right| \leq \mathrm{M} .
\end{gathered}
$$


The elements of the diffusion matrix are continuously differentiable. Therefore, for stochastic differential equation describing typhoid dynamics in (16), we obtain

$$
\|f\|=\sqrt{\sum_{i=1}^{6} f_{i}(x)^{2}} \text { and }\|G\|=\sqrt{\sum_{i=1}^{6} \sum_{j=1}^{19} b_{i j}(x)^{2}} .
$$

Both $\|f\|$ and $\|G\|$ are continuously differentiable and hence satisfy the Lipschitz condition. Since the norms exist, they are bounded. The drift and the diffusion matrices are therefore bounded. Hence, they satisfy the conditions for existence and uniqueness of solution.

\section{Results}

In this section, the behavior of the impact of the parameters involved in the computation of $R_{t y p}\left(R_{t y p} \approx\right.$ $0.7944)$ of the deterministic model (1) is analyzed as shown in Figures 2-9. The parameter values and sources can be seen in Table 1. The following initial starts were adopted;

$$
S_{p}=70, V_{c}=30, I_{f}=20, C_{r}=40, T_{r}=25 .
$$

Also, the stochastic Euler - Maruyama scheme via matlab is used to solve the stochastic model equations (16), and the results of each sub-population of the model are plotted against time, taken to be 15 days.

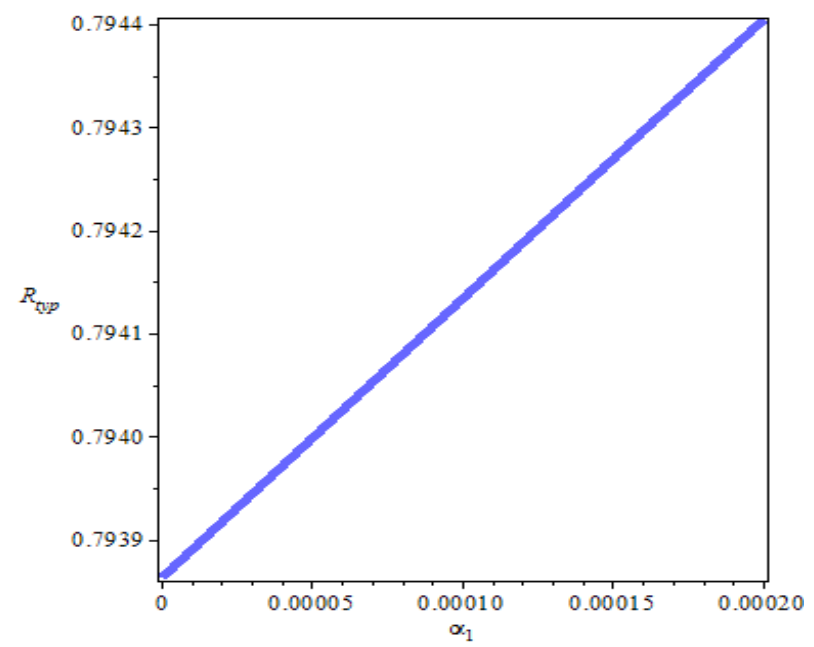

Figure 2. Impact of $\alpha_{1}$ on $R_{t y p}$.

Figure 2 describes the impact of the parameter $\alpha_{1}(0.0020)$ on $R_{t y p}$. The sharp rise of the curve denotes that $R_{t y p}$ is likely to rise above unity when effective infectious contact occurs regularly between typhoid infected and susceptible individuals. To minimize typhoid infections, Healthy measures have to be in place to keep $R_{\text {typ }}<1$.

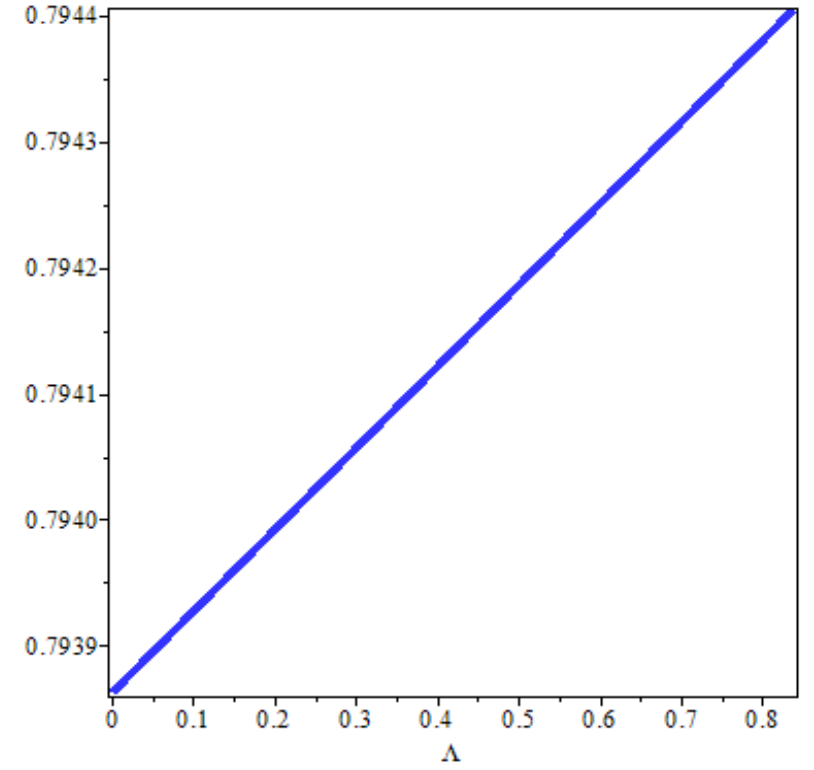

Figure 3. Impact of $\Lambda$ on $R_{\text {typ }}$.

Figure 3 depicts that as birth rate increases, susceptibility to typhoid infection increases which is likely to increase $R_{t y p}$ unless control measures of vaccination are administered to susceptible individuals before being infected with typhoid fever disease.

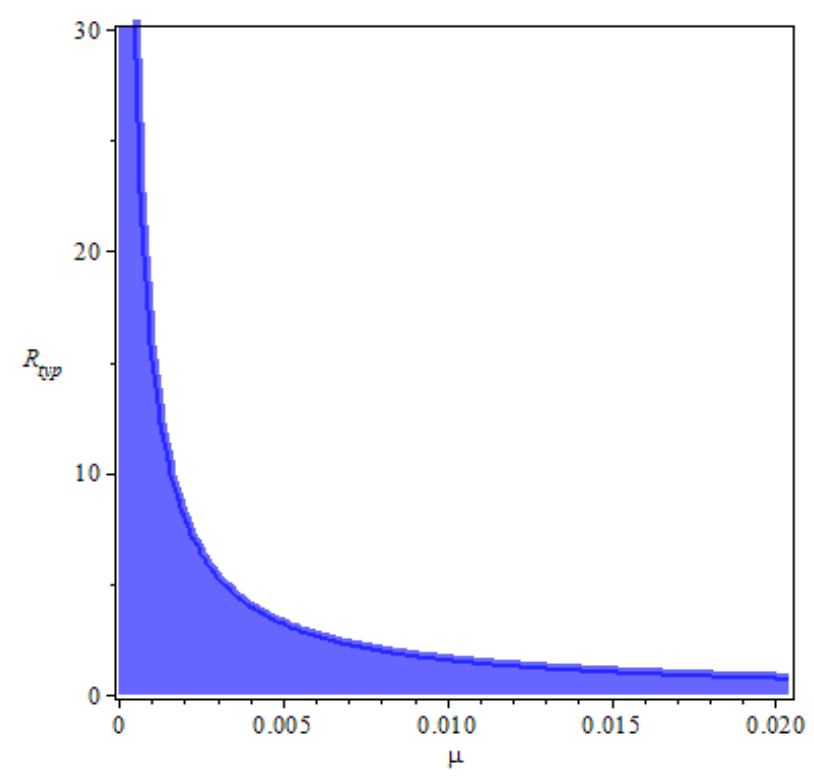

Figure 4. Impact of $\mu$ on $R_{t y p}$.

Figure 4 describes the impact of natural death on $R_{t y p}$. The decline shows that typhoid infection can be minimized by natural death and $R_{t y p}$ is less than 1 . 


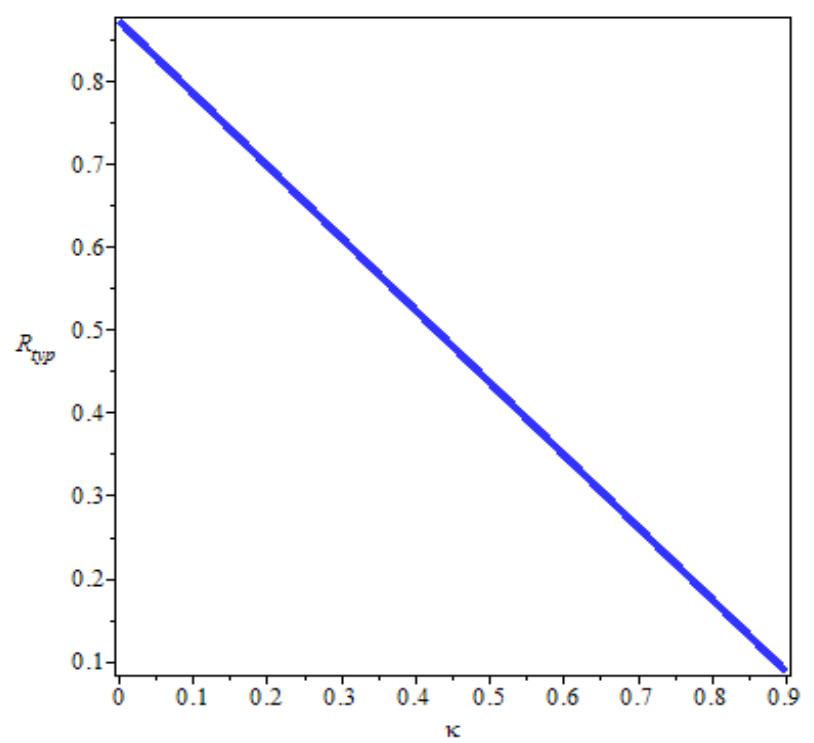

Figure 5. Impact of $\kappa$ on $R_{t y p}$

Figure 5 describes the curve of $\kappa(0.9)$, which denotes the vaccination of proportion of immigrants. This depicts that timely vaccination is a potent strategy in reducing and probably eliminating typhoid in immigrants, where the steady decline of the curve shows that $R_{t y p}$ is less than 1 .

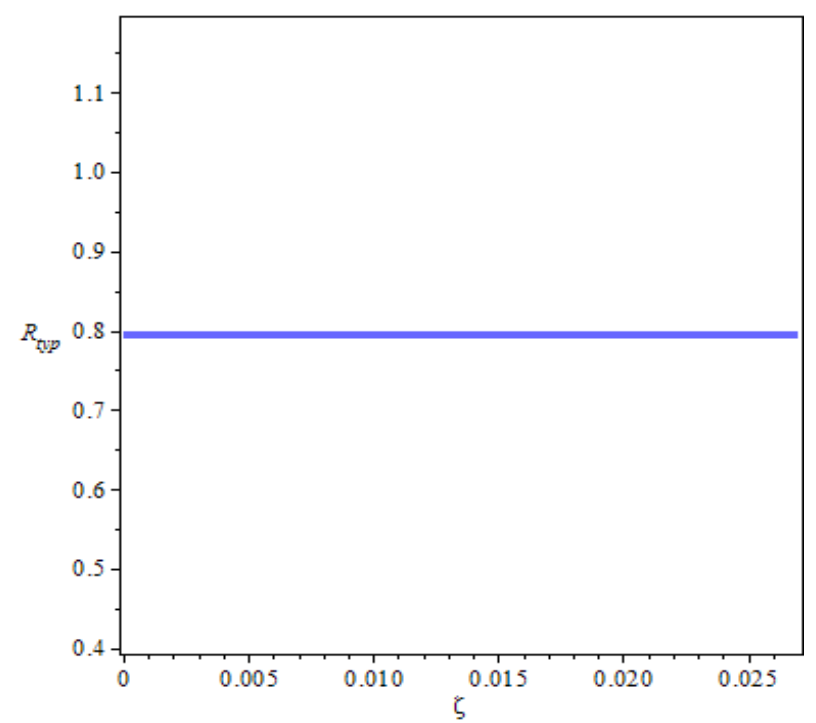

Figure 6. Impact of $\zeta$ on $R_{\text {typ }}$

Figure 6 shows the impact of $\zeta$ on $R_{\text {typ }} . R_{\text {typ }}$ could be greater than 1 , if in the event of steady influx of immigrants, vaccines are not applied timely or not readily available. Therefore, healthy measures must be adhered to by immigrants to probably eliminate typhoid fever in human host community.

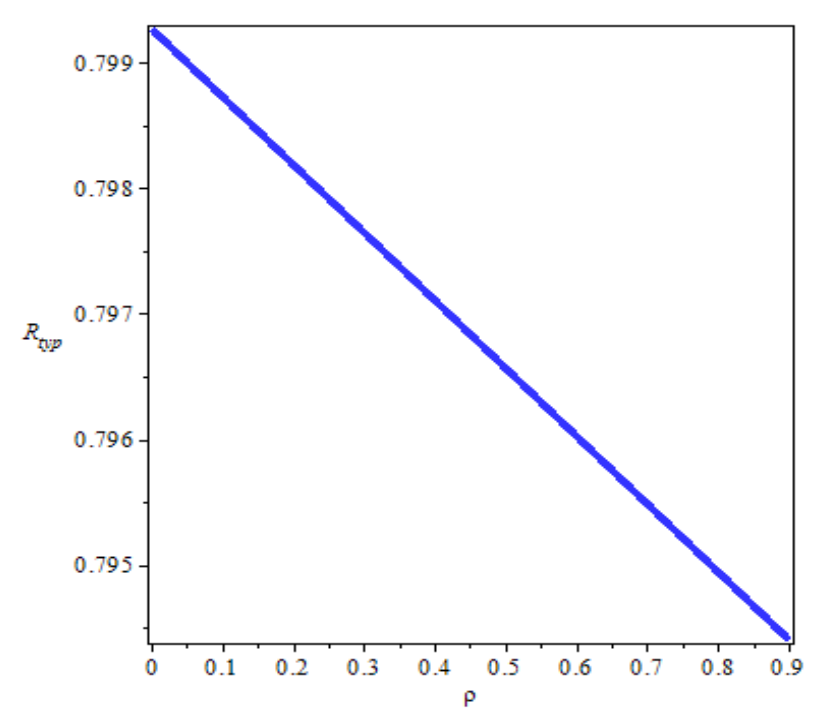

Figure 7. Impact of $\rho$ on $R_{t y p}$.

Figure 7 describes the effect of timely vaccination of susceptible births. The sharp decline shows that early administration of vaccination is effective in controlling $R_{\text {typ }}$ below unity and with consistent vaccination, elimination of typhoid fever in human host community is certain.

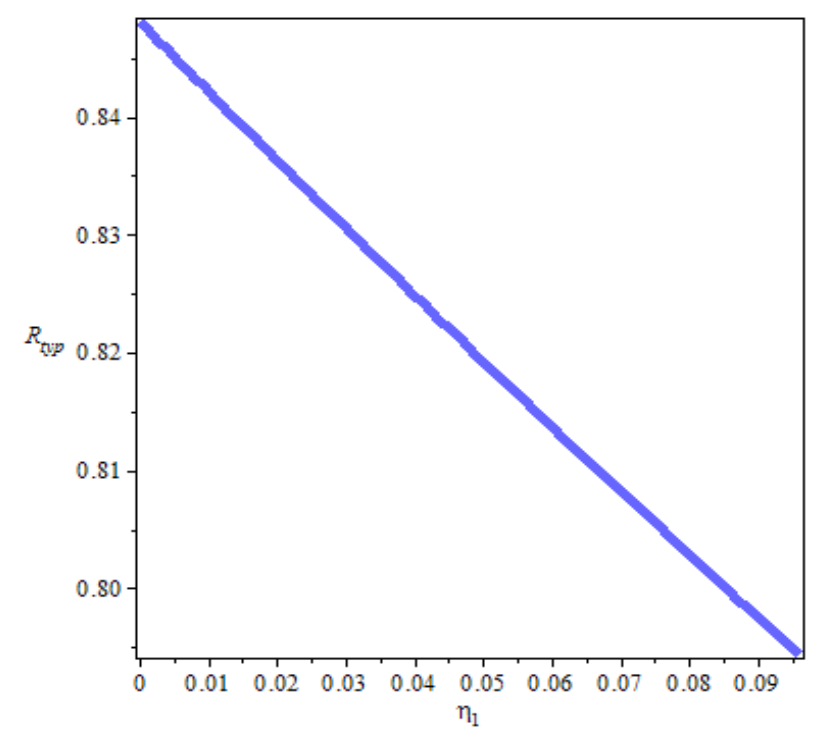

Figure 8. Impact of $\eta_{1}$ on $R_{t y p}$

Figure 8 shows that compliance to timely and effective treatment of typhoid infected individuals, treatment has a positive impact in lessening the basic threshold $R_{t y p}$ below 1. drugs like antibiotics are needed to treat the disease. 


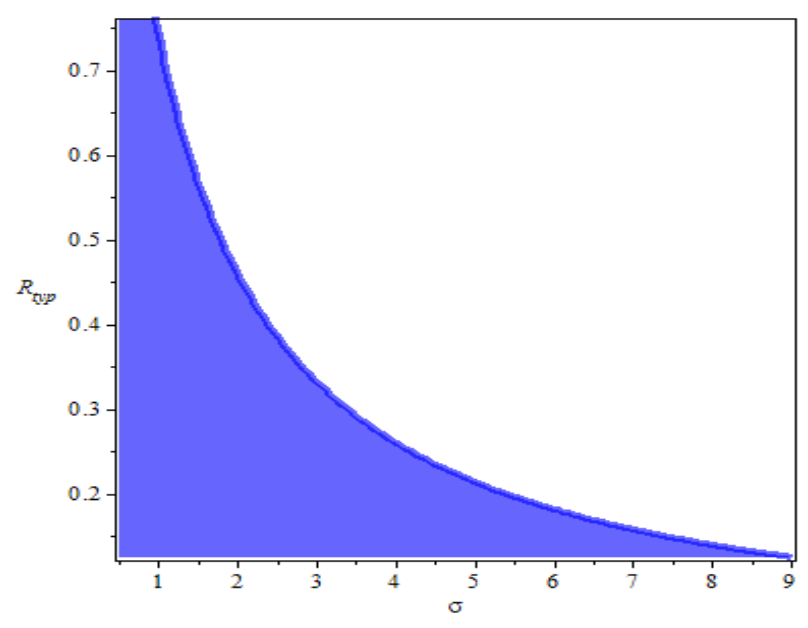

Figure 9. Impact of $\sigma$ on $R_{\text {typ }}$

Figure 9 describes the decline in the curve of $\sigma$ against $R_{\text {typ }}$. This shows that death due to typhoid infection is capable of reducing the value of $R_{t y p}$ below unity, but in other to avoid death due to typhoid, the intervention strategies of screening, treatment and vaccination are to be applied on time to minimize typhoid related death.

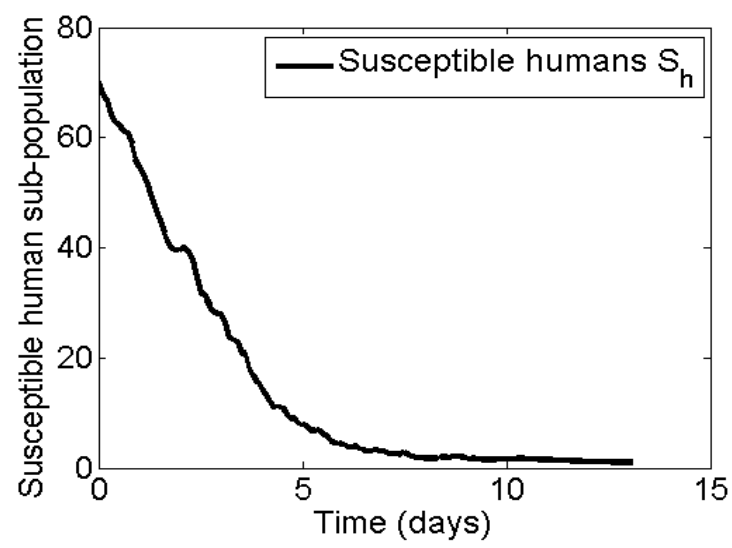

Figure 10. Solution of $S_{p}$ against time (days)

Figure 10 shows that between 5 - 15 days, susceptible sub-population decreases and becomes gradually infected, especially if vaccines are not applied on time or not available. Also, the gradual decline may be due to certain individuals who refuse to be available for vaccine administration, or factors of death due to natural causes.

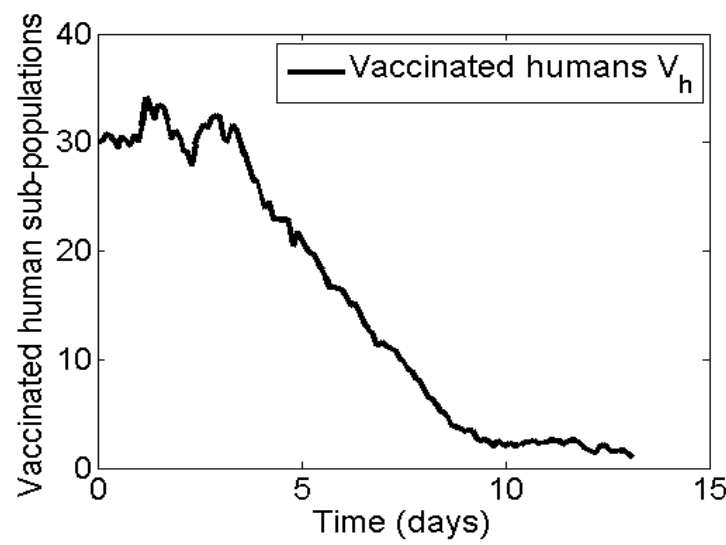

Figure 11. Solution of $V_{c}$ against time (days)

Figure 11 shows that vaccination of susceptible births and immigrants are effective in controlling typhoid disease. The steady decline shows that between $10-15$ days, typhoid infection will be lessened. Therefore, vaccination programs must be made available in the human host community

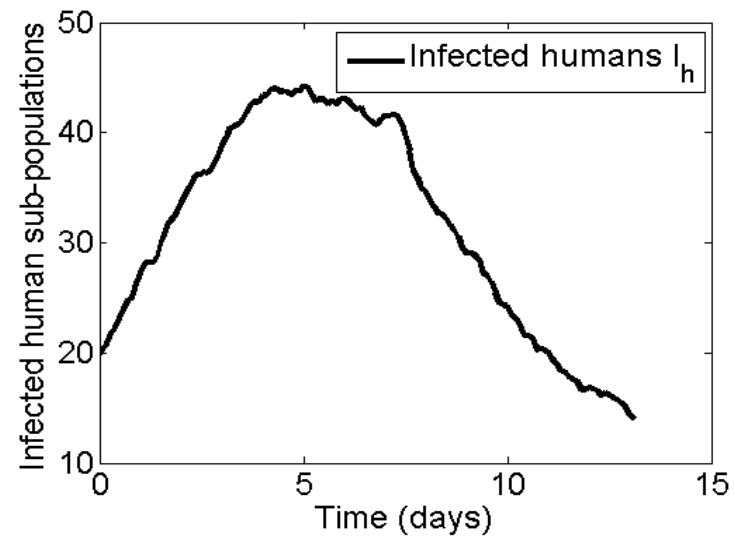

Figure 12. Solution of $I_{f}$ against time (days)

Figure 12 shows that the infection level rose from 20 to about 45 individuals in 5 days, but it suddenly declines due to adherence to screening and treatment of infected individuals. Also, the sudden decline occurs due to death related to typhoid infection. However, treatment is very essential in reducing typhoid fever infection in human host community. 


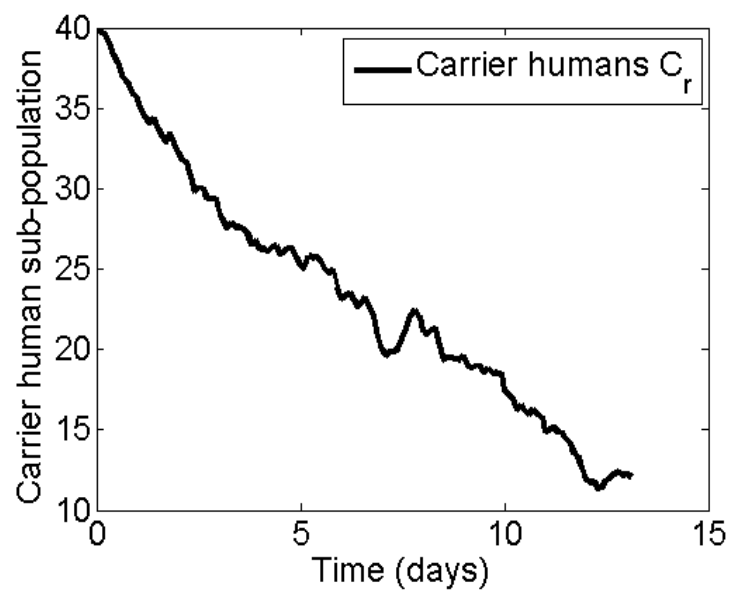

Figure 13. Solution of $C_{r}$ against time (days)

The decline in behavior in Figure 13 depicts a decrease in the carrier sub-population within 15 days. The decrease is due to screening and treatment against typhoid infection.

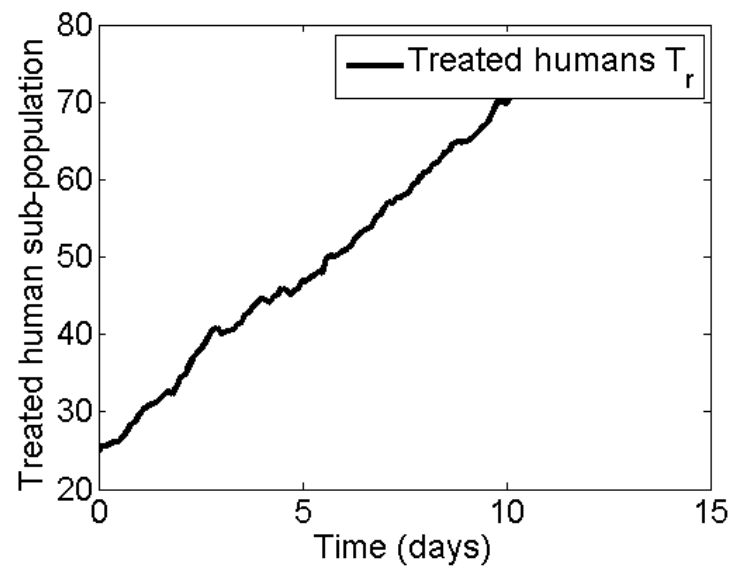

Figure 14. Solution of $T_{r}$ against time (days)

Figure 14 shows the gradual rise of treated individuals within 15 days. Administration of antibiotics is effective in stemming the increase of typhoid fever infection in human host community, leading to a corresponding increase of treated individuals in the host community

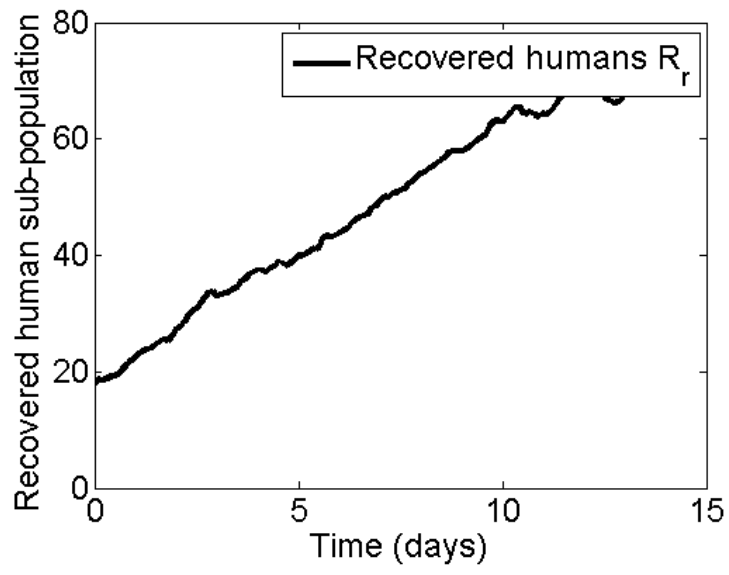

Figure 15. Solution of $R_{r}$ against time (days)
Figure 15 shows the behavior of the solution of recovered populations. The recovered population increases due to response of the treated class to antibiotics treatment against typhoid.

\section{Conclusions}

A deterministic and stochastic model describing the transmission of typhoid is considered. The deterministic Model is shown to be well defined and reasonable in the sense of typhoid epidemic. $R_{t y p}$ is obtained to be less than $1\left(R_{\text {typ }} \approx 0.7944\right)$, which showed that vaccination is very effective in curtailing typhoid in susceptible births and influx of immigrants. The results of the impact of the model parameter on $R_{t y p}$ are graphically displayed and analyzed. The deterministic model is further transformed into a stochastic model by obtaining the drift and diffusion equations. Also, the existence and the uniqueness of the stochastic model are analyzed and approximately solved and the solution of each of the sub-population of the total human host population showed that timely vaccination, treatment and screening are effective in curtailing the spread of typhoid.

\section{Acknowledgements}

We are very grateful to experts for their appropriate and constructive suggestions to improve this manuscript.

\section{REFERENCES}

[1] World Health Organization (WHO). WHO fact sheet on typhoid fever http://www.who.int $>$ typhoidfever. Accessed (2019).

[2] Allen, E.J., Allen, L.J.S., Arciniega, A and Greenwood P. E. Construction of equivalent stochastic differential equation models. Stoch. Anal. Appl., 2008, 26(2): 274-297.

[3] Nkemnole, E.B., Osunkeye, R.O. Stochastic model of some endemic infections: Case study based on the medical records of Gbagada general hospital, Lagos state, Nigeria. J. Stat. Appl., 2016, Pro. 5, No. 2, 265-272. http://dx.doi.org/10.18 576/jsap/050207.

[4] Adetunde, I.A. The mathematical model of the dynamical behaviour of tuberculosis disease in the upper region of the northern part of Ghana: a case study of Baku. Current Research in Tuberculosis, 2008, 1(1): 1-6.

[5] Adetunde, I. A. Mathematical models for the dynamics of typhoid fever in Kassena - Nankana district of upper east region of Ghana. J. Modern Math Stat, 2008, 2, 45-49.

[6] Lauria, D.T., Maskery, B., Poulos, C., and Whittington D. An optimization model for reducing typhoid cases in developing countries without increasing public spending. Vaccine, 2009, 27, 1609 - 1621. 
[7] Mushayabasa, S. Impact of vaccines on controlling typhoid. Journal of modern mathematics and Statistics, 2011, 5(2), 54-59.

[8] Watson C. H., Edmunds W. J. A review of typhoid fever transmission dynamics model and economic evaluation of vaccination. Vaccine, Elsevier, 2015, Volume 33, Supplement 3, pp C42-C54.

[9] Adeboye K. R. and Haruna M. "A mathematical model for the transmission and control of malaria and typhoid co-infection using SIRS approach," Nigeria Research Journal of Mathematics, 2015.

[10] Omame, A. R., Umana, A., Iheonu, N. O., and Chioma, S. O. On the existence of a stochastic model of typhoid fever. Mathematical Theory and Modeling 2015, 5(8), 2015.

[11] Makinde O. D., Getachew T. T., David M. Modelling and Optimal control of typhoid disease with cost-effective strategies. Mathematical and Computational Methods in Medicine, 2017, Volume 2017 |Article ID 2324518 | 16 pages | https://doi.org/10.1155/2017/2324518.
[12] Stephen E., Nkuba N. Modelling of Typhoid Fever with Education, Vaccination and Treatment. Engineering Mathematics, 2016, 1(1): 44 - 52. DOI: 10.11648/j. engmath.20160101.14.

[13] Aji B. S., Aldila D., and Handari B. D. (2019). Modeling the impact of limited treatment resources in the success of typhoid intervention, AIP conference proceedings 2202, 020040, http://doi.org/10.1063/1.5141653 2019.

[14] Moatlhodi K., Gosalamang K. Mathematical Analysis of Typhoid Infection with Treatment. J.Math. Sci. Adv. Appl., 2016, 40, 75-91.

[15] Karundithu J.W., Kimani G., Osman S. Mathematical modeling of typhoid fever disease incorporating unprotected human in spread dynamics, Journal of Advances in Mathematics and Computer Science, 2019, 32(3), 1-11.

[16] Jegede O. O. Modeling the stochastic dynamics of typhoid fever and statistical determination of epidemiological outcomes. Master's dissertation Georgia Southern University, 2019. 